\title{
Microstructures and phase transformation in directionally solidified TiAl-Nb alloys
}

\author{
Fu-qiang Zhang', Xian-fei Ding ${ }^{2}$, Hai Nan', * *Ru-yue You${ }^{4}$, Qiang Wang ${ }^{4}$, Jian-ping $\mathrm{He}^{1}$, Yan-qing Su${ }^{3}$, \\ *Yong-feng Liang ${ }^{1}$, and Jun-pin Lin ${ }^{1}$ \\ 1. State Key Laboratory for Advanced Metals and Materials, University of Science and Technology Beijing, Beijing 100083, China \\ 2. Cast Titanium Alloy R\&D Center, Beijing Institute of Aeronautical Materials, Beijing 100095, China \\ 3. National Key Laboratory for Precision Hot Processing of Metals, Harbin Institute of Technology Harbin, Harbin 150001, China \\ 4. AECC Hunan Aviation Powerplant Research Institute, Zhuzhou 412002, China
}

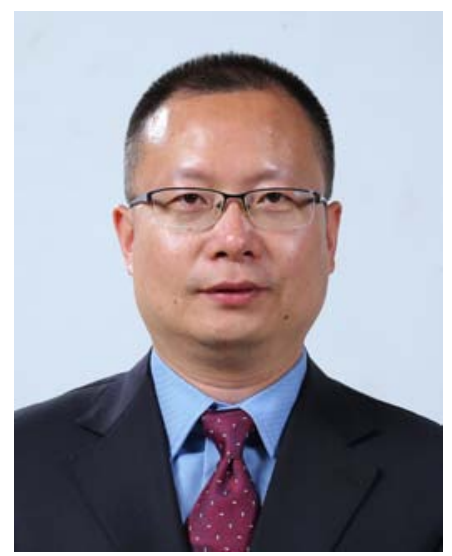

*Yong-feng Liang

Born in 1980, Ph. D, Professor. His research interests mainly focus on structural/functional intermetallics (high temperature TiAl alloys, Fe-Si alloys), advanced fabrication processes for brittle materials, application of advanced metallic materials. To date, he has published more than 60 papers indexed by $\mathrm{SCl}$, granted more than 20 patents. He serves as a council member of Foundry Institution of Chinese Mechanical Engineering Society and a member of Editorial Board of Journal of Netshape Forming Engineering.

E-mail: liangyf@skl.ustb.edu.cn

**Ru-yue You

E-mail: youruyuehit@163.com

Received: 2020-06-22

Accepted: 2020-10-19
Abstract: Intermetallic Ti-45Al-8Nb-(W, B, Y) (at.\%) and Ti-46Al-5Nb alloys are directionally solidified at a constant growth rate of $30 \mu \mathrm{m} \cdot \mathrm{s}^{-1}$ using a Bridgman type apparatus. The quenched microstructures and lengths of different phase regions were observed and measured after various growing times of 0-30 min. Results show that the phase transformations in different phase regions are mainly depending on the high temperature microstructure and the supercooling degree during quenching process. After isothermal holding, the primary phase grows into the liquid phase, the dendrites change from equiaxed to columnar grains, and the length of the $L+\beta$ phase region, $L+\beta+\alpha$ phase region and mushy zone varies, indicating that the entire directional solidification process can be described by a static equilibrium - nonequilibrium dynamic equilibrium evolution process. In addition, the gap between the original growth interface and front interface shows that the actual crystal growth rate is not equal to the drawing velocity during directional solidification.

Key words: TiAl alloys; directional solidification; quenched microstructure; phase transformation

CLC numbers: TG146.23; Document code: A; Article ID: 1672-6421 2020 06-402-07

Tn recent years, TiAl-based alloys containing $\mathrm{Nb}$ were considered the potential candidates for high temperature structural applications due to their excellent tensile strength, good oxidation resistance, and outstanding creep resistance at high temperatures ${ }^{[1-3]}$. However, poor room temperature ductility and hard workability limit their widespread application in aircrafts and vehicles ${ }^{[4]}$. It has been reported that a fully lamellar microstructure with aligned lamellar boundaries, which can be fabricated by directional solidification technology ${ }^{[5]}$, can provide a good balance between the high temperature mechanical properties and ductility at room temperature.

It is difficult to control the lamellar orientation in TiAl-based alloys during directional solidification, because of the complicated solidification process and solid-state phase transformation of the alloys. Ding et al. ${ }^{[7-9]}$ successfully obtained a fully lamellar microstructure with aligned lamellae by a closely-complete peritectic transformation, which can avoid the formation of grains with multiple orientations from the $\beta \rightarrow \alpha$ solid state transformation. Many studies focused on microstructural evolution and microsegregation in the mushy zone ${ }^{[10,11]}$. Few studies can be found focusing on the solid phase structure below the mushy zone. In fact, microstructural evolution is affected not only by the competitive growth and preferred orientation of the front interface in the mushy zone, but also by the high temperature solid phase. Observations of the solid phase region variation are helpful for understanding the phase transformation and microstructural evolution during directional solidification.

In this study, two TiAl-based alloys, Ti-45Al-8Nb-(W, B, Y) and Ti-46Al-5Nb, with 
different solidification paths, were fabricated at a constant drawing velocity using a Bridgman type apparatus. The morphologies of the quenched microstructures in the mushy zone and solid phase regions were observed and analyzed after different growing times. The results are helpful in understanding the phase transformation sequence, to control the microstructure of TiAl alloys during the directional solidification.

\section{Experimental procedure}

The master bars of Ti-45Al-8Nb-(W, B, Y) and Ti-46Al$5 \mathrm{Nb}$ alloys with sizes of $6 \mathrm{~mm}$ in diameter and $120 \mathrm{~mm}$ in length were cut from the induction skull melting ingots, and the oxygen content of both alloys was less than 800 ppm by weight. The chemical compositions of the ingots were Ti45.12Al-8.23 Nb-(W, B, Y) and Ti-45.69Al-5.08Nb (at.\%). After a surface polishing treatment, the bars were placed into the yttria crucibles and melted. A Bridgman type apparatus ${ }^{[9]}$ was used to perform the directional solidification experiments under the protection of $380 \mathrm{~Pa}$ high-purity argon. The TiAl bar in the crucible was held at $1,630{ }^{\circ} \mathrm{C}$ for $20 \mathrm{~min}$ and then directionally solidified under a temperature gradient $(\mathrm{G})$ of $5 \mathrm{~K} \cdot \mathrm{mm}^{-1}$ at a constant drawing velocity of $30 \mu \mathrm{m} \cdot \mathrm{s}^{-1}$. After different drawing times of $0,10,20$, and $30 \mathrm{~min}$, the TiAl bars were quenched in a Ga-In-Sn cooling liquid to preserve the high-temperature microstructure.
The directionally solidified bars were sectioned longitudinally and electropolished in a solution of $5 \%$ perchloric acid, $35 \%$ n-butyl alcohol and 60\% methyl alcohol for microstructural analysis. A ZEISS SUPRA 55 field-emission scanning electron microscope (SEM) with backscattered electron (BSE) mode was used to observe the microstructural morphology and analyze the elemental composition.

\section{Results and discussion}

\subsection{Microstructure of Ti-45Al-8Nb-(W, B, Y) alloy}

Figure 1 shows the longitudinal quenched microstructure of the directionally solidified Ti-45Al-8Nb-(W, B, Y) bars at a growth rate of $30 \mu \mathrm{m} \cdot \mathrm{s}^{-1}$ and different growing times. In Fig. 1(a), the microstructures in different phase regions are marked by A-F. The bright region to the left of Line 1 in Fig. 1(a) exhibits mixed solid and liquid phases, namely mushy zone. It can be speculated that abundant equiaxed dendrites solidified from liquid phase after isothermal holding. The length of the mushy zone can reach a few millimeters. As shown in Fig. 1(b), the morphologies of the microstructures in the mushy zone at a growing time of $10 \mathrm{~min}$ present typical columnar dendrites. It is noted that the mushy zone visibly extends during directional solidification, and the morphology of the front interface changes from plane to dendrite after the directional solidification process begins.

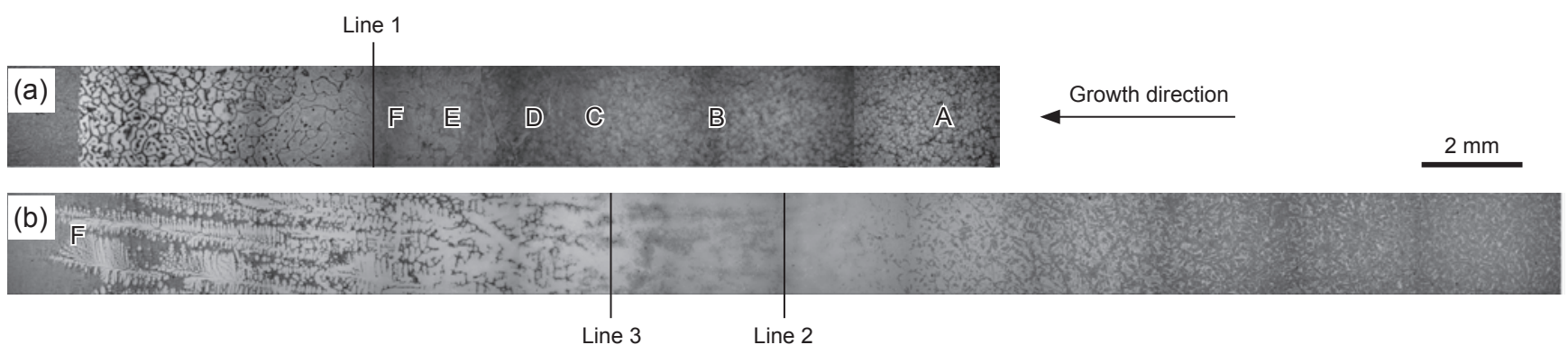

Fig. 1: Longitudinal microstructures of directionally solidified Ti-45AI-8Nb-(W, B, Y) alloy at growing times of $0 \mathrm{~min}(\mathrm{a})$ and $10 \mathrm{~min}(\mathrm{~b})$

Figure 2 shows the quenched microstructures of all the regions from low-temperature to high-temperature liquid phase in directionally solidified Ti-45Al-8Nb-(W, B, Y) alloy. Figure 2(a) shows a mainly lamellar structure with retained B2 and $\gamma$ phases existing at colony boundaries and triple junctions. The bright $\beta$-segregation in the colony is formed from the $\beta \rightarrow \alpha$ solidstate transformation during casting. The region shown in Fig. 2(a) corresponds to Region A in Fig. 1(a), which was subjected to a quite low temperature so that the as-cast microstructure was preserved $^{[12]}$. Figure 2(b) shows a similar microstructure to Fig. 2(a), however, the $\beta$-segregation is distinctly reduced and the colony size is increased. The reason could be that the region shown in Fig. 2(b), corresponds to Region B in Fig. 1(a), subjected to a higher temperature, which led to the increased diffusion of $\mathrm{Nb}$ and $\mathrm{Al}$ and resulted in grain growth and a more homogeneous microstructure. Compared to the ascast microstructure in Region A, the microstructure in Region
B changes a little, so Region B is called the annealing region. The microstructures quenched from $\alpha+\gamma$ and $\alpha$ phase regions [Regions C and D in Fig. 1(a)] are shown in Figs. 2(c) and (d), respectively. After quenching from the $\alpha+\gamma$ two-phase region, the microstructures consisting of lamellar colonies with large lamellar spacing and massive $\gamma$ grains were transformed from $\alpha$ phase, along with little retained B2 phase ${ }^{[13,14]}$. It is obvious that the lamellar spacing in Region $C$ is greater than that in Region $\mathrm{B}$, because of the lamellar spacing increases as the diffusion rate of the $\mathrm{Al}$ solute increases ${ }^{[15,16]}$. As the temperature increases, more $\gamma$ plates transform to $\alpha$ plates, which results in a greater lamellar spacing and more equiaxed $\alpha$ grains. The lamellar structure disappears when the temperature reaches the $\alpha$ singlephase region. It is difficult to observe the lamellar colonies in Fig. 2(d), indicating that Region D is located in the $\alpha$ singlephase region. It can be speculated that lamellar structure is retained in the $\alpha+\gamma$ two-phase region but disappears in the $\alpha$ 
single-phase region. The locations of $\alpha+\gamma$ and $\alpha$ phase regions in the sample can be determined by observing the quenched lamellar structure. As shown in Fig. 2(e) [Region E in Fig. 1(a)], the gray areas present thick $\alpha$ laths, the white irregular $\beta$-segregation distributes in $\alpha$ laths, and the dark areas beside $\alpha$ laths are quenched massive $\gamma$ grains. The $\beta$-segregation is formed during the $\beta \rightarrow \alpha$ solid-state transformation. Note that the $\mathrm{Nb}$ element prefers to distribute in $\beta$ phase rather than $\alpha$ and $\gamma$ phases. When $\alpha$ nucleates in $\beta$, the $\mathrm{Nb}$ is rejected and forms the irregular network segregation. Figure 2(f) shows the quenched microstructure from the $\beta$ single-phase region [Region F in Fig. 1(a)]. Large amounts of $\alpha$ phase appear as plates surrounded by $\beta$-segregation. No massive $\gamma$ is observed in Fig. 2(f), which demonstrates that Region E locates in the $\alpha+\beta$ two-phase region and Region $F$ locates in the $\alpha$ singlephase region. Similar to the quenched lamellar structure, the
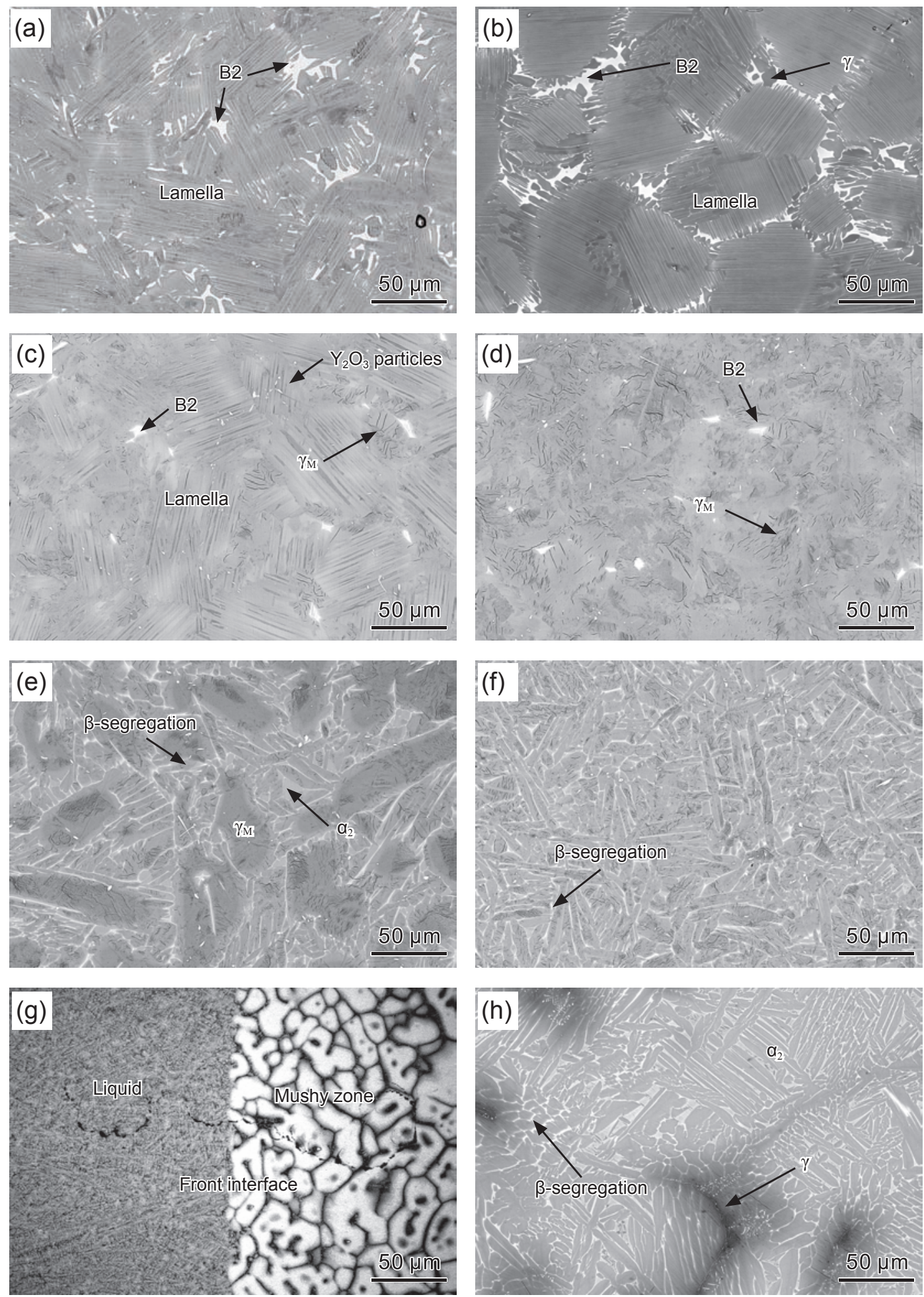

Fig. 2: Microstructural morphologies of different phase regions in directionally solidified Ti-45Al-8Nb- (W, B, Y) alloy at a growing time of 0 min: (a) as-cast region; (b) annealing region; (c) $\alpha+\gamma$ region; (d) $\alpha$ region; (e) $\alpha+\beta$ region; (f) $\beta$ region; (g) mushy zone; (h) high-magnification image of mushy zone 
massive $\gamma$ can be used to distinguish $\alpha+\beta$ and $\beta$ phase regions. Figures $2(\mathrm{~g})$ and $2(\mathrm{~h})$ show the morphologies of the quenched mushy zone. The bright island-like regions represent equiaxed $\alpha$ phase, transformed from the high-temperature $\beta$ phase in the mushy zone during quenching. The dark regions represent the $\gamma$ phase transformed from the interdendritic liquid, which surrounds the $\beta$ phase at high temperature. Figure 2(h) shows the concentrated $\beta$-segregation distributed in quenched $\alpha$ phase, just like that in Fig. 2(f). The mushy zone corresponds to $\beta+\mathrm{L}$ phase region.

Figure 3 shows the quenched microstructural morphologies of the interfaces between the different phase regions. Figure 3(a) shows the interface marked by Line 1 in Fig. 1(a), where the dotted line indicates the interface between the $\beta+\mathrm{L}$ phase region (mushy zone) and the $\beta$ single-phase region. To obtain the quenched microstructures in Fig. 2 and Fig. 3(a), the bars must be quenched immediately after the isothermal holding. Figure 3(c) shows the interface between $\beta+\mathrm{L}$ phase region and $\beta$ phase region, which corresponds to the region indicated by Line 3 in Fig. 1(b). The solid phase in the two-phase region shows the columnar dendritic morphology, which is distinct from that in Fig. 3(a). The dotted line in Fig. 3(b) shows the original growth interface corresponds to the region indicated by Line 2 in Fig. 1(b). The region to the right of the dotted line was actually the mushy zone $10 \mathrm{~min}$ earlier, and the region to the left of the dotted line is the growth region. As described above, the morphology of the front interface changes from plane to dendrite after the directional solidification process starts. The columnar dendrite in the growth region is evident, which demonstrates the crystal growth originates from the region to the left of the dotted line in Fig. 2(b). That region is actually the mushy zone 10 min earlier, thus it is called the original mushy zone, and the interface between the original mushy zone and the growth region is the original growth interface. Noticeable Al segregation in the interdendritic region is observed in the growth region marked in Fig. 3(b). The reason could be that directional solidification is a nonequilibrium process. The nonequilibrium phase transformation occurs in the mushy zone, and the growth of primary $\beta$ phase results in the segregation of $\mathrm{Al}$ in the interdendritic liquid, and $\mathrm{Nb}$ in the dendritic cores, owing to the difference in the element distribution coefficients ${ }^{[17]}$. As shown in Fig. 3(c), the dotted line represents the interface between the $L+\beta$ two-phase region and $\beta$ single-phase region. The $\mathrm{L}+\beta$ two-phase region is actually the mushy zone, and the $\beta$ single-phase region is in essence the growth region. The Al segregation is more distinct than that in the Fig. 3(b), which is caused by the higher supercooling. It can be summarized that two interfaces connect the mushy zone, growth region, and original mushy zone. Figure 3(d) shows the quenched front interface morphology at a growing time of $10 \mathrm{~min}$. In contrast to Fig. 2(g), the solid phase is columnar dendrites, and the angle between the primary and secondary dendrite arms is $90^{\circ}$, which demonstrates that the primary phase is the $\beta$ phase.
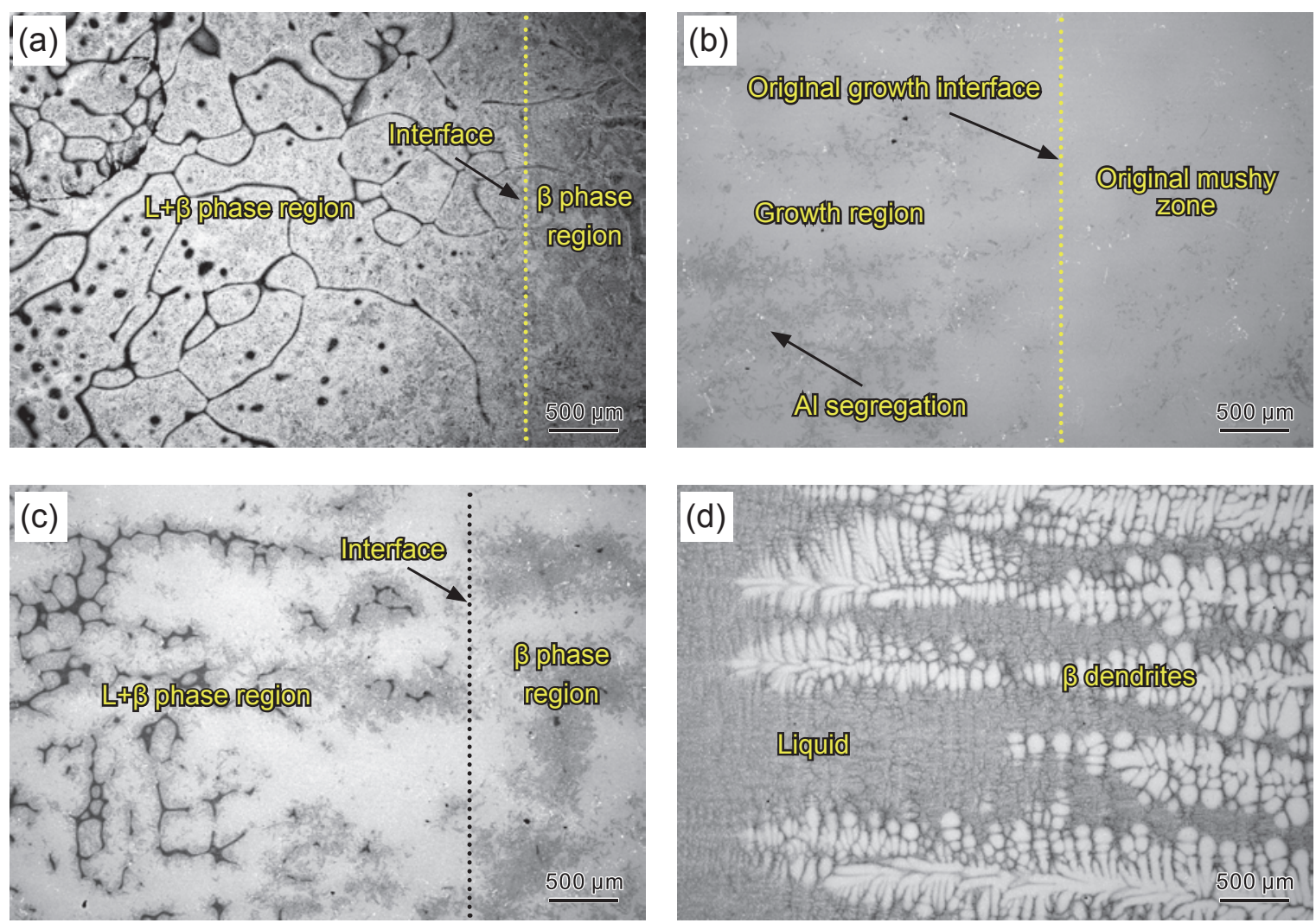

Fig. 3: Microstructures at different interfaces of directionally solidified Ti-45AI-8Nb-(W, B, Y) alloy: (a) interface of $L+\beta / \beta$ with a growing time of $0 \mathrm{~min}$; (b) original growth interface with a growing time of $10 \mathrm{~min}$; (c) interface of $L+\beta / \beta$ with a growing time of $10 \mathrm{~min}$; (d) front interface with a growing time of $10 \mathrm{~min}$ 


\subsection{Microstructure of Ti-46Al-5Nb alloy}

Figure 4 shows the BSE micrographs of the growth region during directional solidification for the Ti-46Al-5Nb alloy at a growing time of $10 \mathrm{~min}$. As shown in Fig. 4(a), the original growth interface is labeled by Line 2 . The region below Line 1 is the original mushy zone and the primary phase grows into the liquid from this region after the directional solidification process starts. Line 1 divides the $\beta+\alpha$ and $L+\beta+\alpha$ phase regions. The region between Line 1 and Line 2 is the growth region. It can be found that the growth region shows a dendritic morphology without a consistent orientation. Figure 4(b-d) shows the highmagnification microstructures of different phase regions marked by A-C in Fig. 4(a), respectively. Figure 4(b) shows the microstructures quenched from the $\mathrm{L}+\beta$ two-phase region, which consist of the $\alpha$ phase at dendritic cores and $\gamma$ phase at interdendritic areas. The primary phase can be determined as $\beta$ phase by observing the $\beta$-segregation distributed in $\alpha$ dendrites and the angle between the primary dendrite trunk and the secondary dendrite arm. The interdendritic $\gamma$ phase is transformed from the liquid during quenching. Figure 4(c) shows the quenched morphology of the $\mathrm{L}+\beta+\alpha$ three-phase region, and peritectic transformation should proceed in this region ${ }^{[9]}$. The bright gray $\alpha$ phase in the dendritic core region accompanied by $\beta$-segregation is transformed from $\beta$ phase during quenching, while there is no $\beta$-segregation in the gray $\alpha$ phase in the edge of the dendritic arm, revealing that peritectic transformation occurs in this region. As the temperature decreases, the $\alpha$ phase nucleates around the $\beta$ dendritic arms, and grows toward the dendritic core and liquid simultaneously until one of them is exhausted. The yttria particles located in

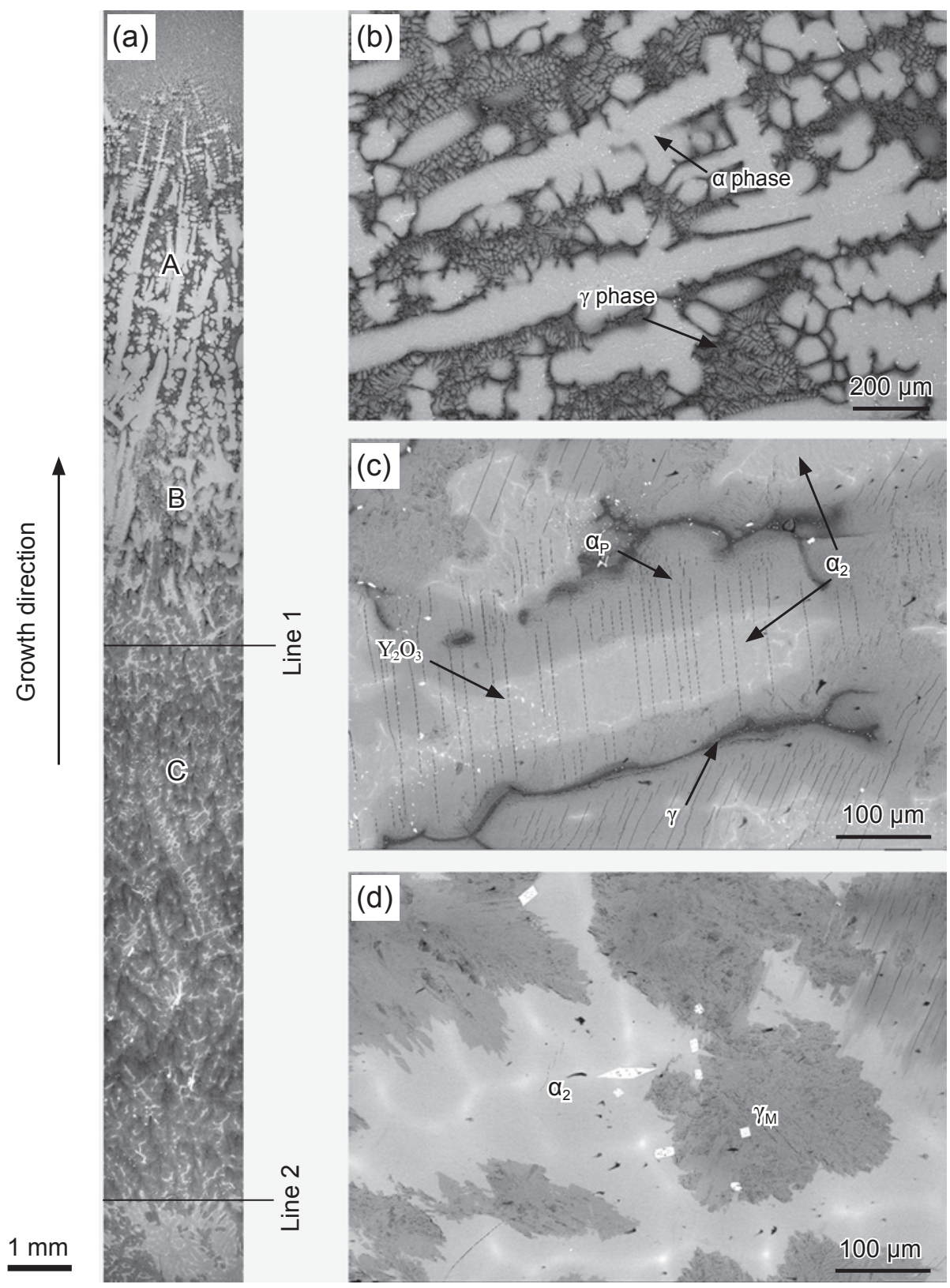

Fig. 4: Microstructures of directionally solidified Ti-46Al-5Nb alloy: (a) longitudinal microstructure at a growing time of $10 \mathrm{~min}$; (b) quenched morphology of $L+\beta$ region; (c) quenched morphology of $L+\beta+\alpha$ region;

(d) quenched morphology of $\beta+\alpha$ region 
the dendritic core and interdendritic regions are released into the melt because of the erosion of the crucible by heat flow. The microstructural morphology in Fig. 4(d) is similar to that shown in Fig. 2(e). The $\beta$ phase transforms into $\alpha$ phase, and the $\alpha$ phase transforms into massive $\gamma$ phase. However, the $\beta$-segregation in $\alpha$ phase shown in Fig. 4(d) is slighter than that in Fig. 2(e), perhaps due to the different $\mathrm{Nb}$ concentrations in the two alloys, higher $\mathrm{Nb}$ leads to more distinct $\beta$-segregation.

\subsection{Phase transformation and phase region analysis}

Directional solidification is a typical nonequilibrium phase transformation process. Differences in the supercooling temperature lead to the diversity of the quenched microstructures. In addition, the solidification path of the alloy and the phase transformation during solidification affect the quenched microstructural morphology. As discussed above, different quenched microstructures are obtained for different phase regions. The high-temperature microstructures can be speculated by observing quenched microstructures. For a directionally solidified alloy, the locations and lengths of different phase regions, especially the high-temperature phase regions, can be measured by distinguishing the quenched microstructures. In general, the dendrite grows into liquid and eventually reaches a stable state. After the isothermal holding, the growth of the primary phase in the front interface disturbs the previously established balance. The mass and heat transformations should change to achieve a new dynamic equilibrium during directional solidification. The dynamic equilibrium extent of the whole directional solidification process can be delivered by the variation of those phase regions. The microstructural evolution can be traced via analyzing the quenched microstructure and the corresponding phase regions. The statistical results of the different phase regions in the directionally solidified $\mathrm{Ti}-46 \mathrm{Al}-5 \mathrm{Nb}$ alloy at different growing times are summarized in Table 1.

Table 1: Length of phase region in directionally solidified Ti-46Al-5Nb alloy at different growing times

\begin{tabular}{|c|c|c|c|c|}
\hline \multirow{2}{*}{ Phase region } & \multicolumn{4}{|c|}{ Length (mm) } \\
\hline & $0 \mathrm{~min}$ & $10 \mathrm{~min}$ & $20 \mathrm{~min}$ & $30 \mathrm{~min}$ \\
\hline$L+\beta$ & 3.22 & 4.57 & 4.62 & 4.79 \\
\hline$L+\beta+\alpha$ & 1.85 & 2.16 & 2.84 & 3.28 \\
\hline$\beta+\alpha$ & 3.69 & - & - & - \\
\hline
\end{tabular}

Figure 5 shows the lengths of $\mathrm{L}+\beta$ phase region and $\mathrm{L}+\beta+\alpha$ phase region of $\mathrm{Ti}-46 \mathrm{Al}-5 \mathrm{Nb}$ alloy, along with the theoretical and measured distances between the original growth interface and the front interface. The lengths of the $L+\beta$ and $L+\beta+\alpha$ phase regions increase with an increase in growing time, and dramatic variation in the $L+\beta$ phase region occurs in the first 10 min during directional solidification. In contrast, the change

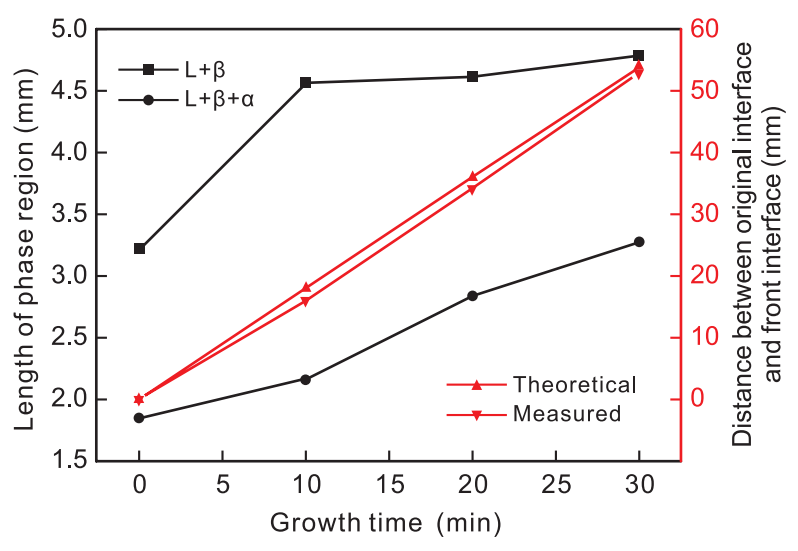

Fig. 5: Length of different regions and theoretical and measured distances between original growth interface and front interface at various growing times of Ti-46Al-5Nb alloy

in the $\mathrm{L}+\beta+\alpha$ peritectic phase region is stable throughout the directional solidification. Generally, the drawing velocity of the directionally solidified bar is equal to the growth rate of the front interface, and the length of drawing should be equal to the distance that the front interface moves forward into the melt. In this work, the drawing velocity is constant at $30 \mu \mathrm{m} \cdot \mathrm{s}^{-1}$, therefore, the distances between the solidi/liquid front interface and the original growth interface should be 18,36 , and $54 \mathrm{~mm}$ after growing times of 10, 20, and $30 \mathrm{~min}$, respectively. However, the measured distances between the $\mathrm{S} / \mathrm{L}$ front interface and the original growth interface are 15.98, 34.24, and $53.02 \mathrm{~mm}$ at growing times of 10, 20, $30 \mathrm{~min}$, less than the theoretical values, as shown in Fig. 5. The gap between the theoretical value and actual value decreases gradually as the directional solidification proceeds. Therefore, it can be inferred that the growth rate of the front interface increases during directional solidification.

\section{Conclusions}

In the present study, quenched microstructures of Ti-45Al$8 \mathrm{Nb}-(\mathrm{W}, \mathrm{B}, \mathrm{Y})$ and Ti-46Al-5Nb alloys directionally solidified at the growth rate of $30 \mu \mathrm{m} \cdot \mathrm{s}^{-1}$ for different times were investigated. The following conclusions can be obtained:

(1) The quenched microstructures of the directionally solidified alloys with different solidification paths are identified. The primary solidification phases are $\beta$ phase for both alloys, while peritectic transformation only occurs for Ti-46Al-5Nb alloy. The front interface morphology changes from plane to dendrite during directional solidification. For Ti-45Al-8Nb(W, B, Y) alloy, the locations of different phase regions in directionally solidified bars are determined. The front interface morphology changes from plane to dendrite during directional solidification.

(2) The measured front interface movement is consistent to the theoretical dendrite growth rate, and a little less than the drawing speed. The growth rate of the front interface increases during the directional solidification process.

(3) The measured front interface movement distance is 
consistent to the theoretical dendrite growth rate, and a little less than the drawing speed. The growth rate of the front interface increases linerly with the time during the directional solidification, while the length of the $L+\beta$ two-phase region increases dramatically in the first $10 \mathrm{~min}$, and then increases slowly.

\section{Acknowledgements}

This research was financially supported by the National Natural Science Foundation of China (Nos.: 51671026, 51831001, and 51921001) and the Fundamental Research Funds for the Central Universities (No. FRF-GF-19-024B).

\section{References}

[1] Appel F, Wagner R. Microstructure and deformation of twophase $y$-titanium aluminides. Materials Science and Engineering R Reports, 1998, 22(5): 187-268.

[2] Clemens H, Mayer S. Design, processing, microstructure, properties, and applications of advanced intermetallic TiA alloys. Advanced Engineering Materials, 2013, 15(4): 191-215.

[3] Lin J P, Xu X J, Wang Y I, et al. High temperature deformation behaviors of a high $\mathrm{Nb}$ containing TiAl alloy. Intermetallics, 2007, 15(5-6): 668-674.

[4] Xu X J, Lin J P, Wang Y I, et al. Effect of forging on microstructure and tensile properties of Ti-45Al-(8-9) Nb-(W, B, Y) alloy. Journal of Alloy \& Compounds, 2006, 414(1-2): 175-180.

[5] Yamaguchi M, Johnson D R, Lee H N, et al. Directional solidification of TiAl-based alloys. Intermetallics, 2000, 8: 511517.

[6] Chen G, Peng Y B, Zheng G, et al. Polysynthetic twinned TiAl single crystals for high-temperature applications. Nature Materials, 2016, 15(8): 876-81.
[7] Ding X F, Lin J P, Zhang L Q, et al. Lamellar orientation control in a Ti-46Al-5Nb alloy by directional solidification. Scripta Materialia, 2011, 65(1): 61-64.

[8] Ding X F, Lin J P, Zhang L Q, et al. Microstructural control of TiAl-Nb alloys by directional solidification. Acta Materialia, 2012, 60(2): 498-506.

[9] Ding X F, Lin J P, Zhang L Q, et al. Microstructure development during directional solidification of Ti-45Al-8Nb alloy. Journal of Alloys \& Compounds, 2010, 506: 115-119.

[10] Luo L S, Liu T, Li K, et al. Microstructures, micro-segregation and solidification path of directionally solidified Ti-45Al-5Nb alloy. China Foundry, 2016, 13(2): 107-113.

[11] Wang Y Z, Ding H S, Chen R R, et al. Dependency of microstructure and microhardness on withdrawal rate of Ti43Al-2Cr-2 Nb alloy prepared by electromagnetic cold crucible directional solidification. China Foundry, 2016, 13(4): 289-293.

[12] Yang G, Kou H C, Liu Y, et al. Response of the solidification microstructure of a high $\mathrm{Nb}$ containing $\mathrm{TiAl}$ alloy to an isothermal high-temperature heat treatment. Intermetallics, 2015, 63: 1-6.

[13] Denquin A, Naka S. Phase transformation mechanisms involved in two-phase TiAl-based alloys - discontinuous coarsening and massive-type transformation. Acta Materialia, 1996, 44: 353-365.

[14] Hu D W, Huang A J, Wu X H. On the massive phase transformation regime in TiAl alloys: the alloying effect on massive/lamellar competition. Intermetallics, 2007, 15(3): 327-332.

[15] Kim Y W. Microstructural evolution and mechanical properties of a forged GAMMA titanium aluminum alloy. Acta Materialia, 1992, 40: 1121-1134.

[16] Tang J C, Huang B Y, Zhou K C, et al. Factors affecting the lamellar spacing in two-phase TiAl alloys with fully lamellar microstructures. Materials Research Bulletin, 2001, 36: 17371742

[17] Liu G H, Li X Z, Su Y Q, et al. Microstructure, microsegregation pattern and the formation of B2 phase in directionally solidified Ti-46Al-8Nb alloy. Journal of Alloys \& Compounds, 2012, 541: 275-282. 